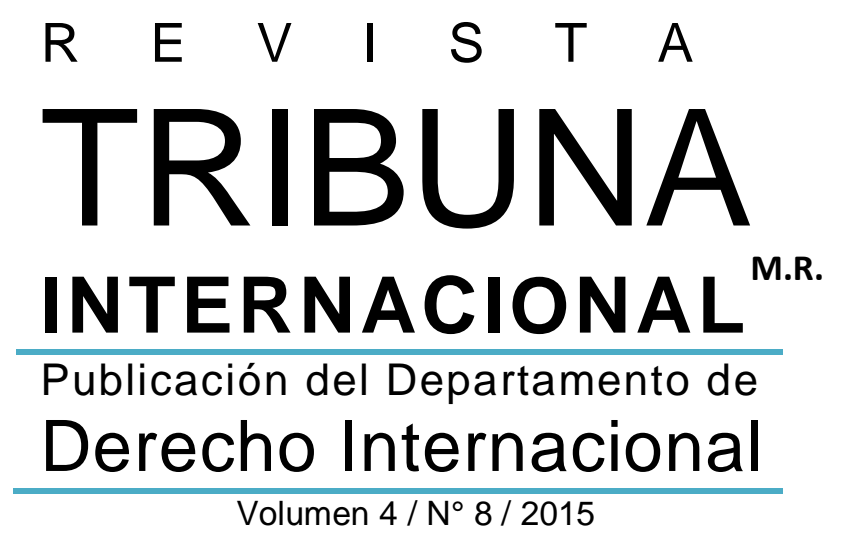

FACULTAD DE DERECHO 


\section{Rector de la Universidad de Chile}

Ennio Vivaldi Véjar

Av. Alameda Libertador Bernardo O'Higgins 1058, Santiago

\section{Representante legal}

Davor Harasic Yaksic

Decano de la Facultad de Derecho

Universidad de Chile

\section{Director Departamento Derecho Internacional}

Edmundo Vargas Carreño

\section{Director (S) Revista Tribuna Internacional}

Mario Arnello Romo

\section{Editor General Revista Tribuna Internacional}

Luis Valentín Ferrada Walker

\section{Comité Editorial}

Íñigo Álvarez Gálvez (Universidad de Chile, Chile)

Gonzalo Aguilar (Universidad de Talca, Chile)

José Carlos Fernández Rosas (Universidad

Complutense de Madrid, España)

Claudio Grossman (American University, EE.UU.)

Mattias Kumm (New York University, EE.UU.)

Hugo Llanos (Universidad Central, Chile)

Cecilia Medina (Universidad Diego Portales, Chile)

Elina Mereminskaya (Universidad de Chile, Chile)

Mónica Pinto (Universidad de Buenos Aires, Argentina)

\section{Fundador de la Revista Tribuna Internacional}

Mario Ramírez Necochea $†$

\section{Revista Tribuna Internacional M.R.}

Publicación del Departamento de Derecho Internacional de la Facultad de Derecho de la Universidad de Chile. Su objetivo es fomentar la reflexión, el debate, el análisis y la comunicación sobre el derecho internacional en forma pluralista y con rigor científico. Se publica cada semestre en los meses de junio y diciembre mediante convocatoria abierta a la publicación de artículos y monografías inéditos, comentarios de jurisprudencia, recensiones y comentarios de libros, en los campos de derecho internacional público y privado, derecho internacional de los derechos humanos y relaciones internacionales, tanto en castellano como inglés.

La Revista Tribuna Internacional fue creada por Decreto Exento No 8.466 de la Rectoría de la Universidad de Chile, de 22 de marzo del 2011

Volumen 4/ No 8 / 2015

www.tribunainternacional.uchile.cl ISSN 0719-482X (versión en línea)

Departamento de Derecho Internacional Facultad de Derecho Universidad de Chile Av. Santa María 076, $4^{\circ}$ piso Providencia, Santiago de Chile

\section{Diseño y producción:}

Facultad de Derecho

Universidad de Chile

Se autoriza la reproducción total o parcial del contenido de la publicación, siempre que se reconozca y cite el/ la/ los/ las autor/a/es/as y la publicación, no se realicen modificaciones a la obra y no se la utilice para fines comerciales. 


\title{
Decisión del Tribunal Especial para El Líbano sobre la admisibilidad en juicio de documentos publicados en el sitio web WikiLeaks ${ }^{1}$
}

Decision of the Special Tribunal for Lebanon on the admissibility in trial of documents disclosed at the WikiLeaks website

\author{
Juan Francisco Lobo Fernández \\ juanfranciscolobo@gmail.com
}

Abogado, Universidad de Chile. Profesor invitado de Jurisdicción Penal Internacional (Universidad de Chile). Coordinador Académico, Cursos de Derechos Humanos Online (UDP). Profesor de Teoría del Derecho (UDP). Profesor de Derecho Penal Internacional (UAI).

Resumen: Luego de analizar la naturaleza excepcional del tribunal híbrido conocido como Tribunal Especial para El Líbano constituido en 2007, se comenta una de sus más recientes sentencias, dictada el 21 de mayo de 2015, en la que deniega la admisibilidad en juicio de ciertos documentos publicados en el sitio web "WikiLeaks". Finalmente se evalúa esta decisión desde una perspectiva de derechos humanos.

Palabras clave: Tribunales híbridos - Tribunal Especial para El Líbano - Prueba - WikiLeaks.

\begin{abstract}
After analyzing the exceptional nature of the bybrid court known as Special Tribunal for Lebanon established in 2007, one of its most recent rulings issued on 21 May 2015 is commented, whereby it denies the admissibility in trial of documents published in the website "WikiLeaks". Finally, this ruling is appraised from a buman rights perspective.
\end{abstract}

Keywords: Hybrid courts - Special Tribunal for Lebanon - Proof - WikiLeaks.

Comentario jurisprudencial enviado el 09.09.2015 y aceptado el 13.12.2015. 


\section{Introducción}

El 14 de febrero de 2005 fue perpetrado un atentado con bomba en el centro de Beirut, la capital de El Líbano. El ataque tuvo como resultado la muerte de 23 personas, incluyendo al ex Primer Ministro libanés Rafiq Hariri.

Como consecuencia de este hecho, el 23 de enero de 2007 el Estado soberano de El Líbano celebró un tratado internacional con la Organización de las Naciones Unidas para la conformación de un tribunal especial para la investigación y juzgamiento de estos y otros hechos calificados como terroristas bajo el derecho penal libanés. Mediante la Resolución $\mathrm{N}^{\circ}$ 1.757 de 30 de mayo de 2007, el Consejo de Seguridad de las Naciones Unidas estableció el Tribunal Especial para El Líbano.

Se trata de un tribunal penal internacionalizado o de aquellos conocidos como "tribunales híbridos". Los tribunales híbridos se caracterizan por aplicar tanto derecho penal doméstico como disposiciones de derecho penal internacional. Asimismo, suelen operar con un componente orgánico internacional, o cuando menos dicho elemento se presenta en su génesis, usualmente con la participación de la Organización de las Naciones Unidas.

Este tipo de tribunales ha proliferado en contextos de justicia transicional a comienzos del siglo XXI. En efecto, en 2000 se constituyeron los Paneles Especiales para Graves Crímenes en Timor Oriental. En 2001 Camboya negoció con las Naciones Unidas para incorporar a su sistema judicial un grupo de Cámaras Extraordinarias. En 2002 Sierra Leona celebró un tratado con las Naciones Unidas para establecer el Tribunal Especial para dicho país. En 2005 se incorporó una Sección Especial para Crímenes de Guerra en la Corte de Bosnia y Herzegovina ${ }^{2}$. Luego del establecimiento del Tribunal Especial para El Líbano, en 2007, por acuerdo entre la Unión Africana y Senegal se crearon en 2013 las Cámaras Africanas Extraordinarias, las que fueron integradas al sistema judicial de este país para ejercer jurisdicción universal sobre hechos cometidos en Chad en la década de $1980^{3}$.

Entre las ventajas de estos tribunales de nuevo cuño se señala que no son tan costosos como los tribunales internacionales (v.g. el Tribunal Penal Internacional para la ex Yugoslavia o el Tribunal Penal Internacional para Ruanda) y que poseen mayor cercanía con los hechos que aquellos, por lo que es menos probable que sean vistos como una imposición foránea. Al mismo tiempo, superan a los tribunales completamente domésticos en situaciones de justicia transicional en que muchos poderes y servicios estatales -incluyendo la judicatura- han cesado de funcionar total o parcialmente ${ }^{4}$.

CASSESE, Antonio et al. International Criminal Law. $3^{\text {a }}$ edición. Oxford, Oxford University Press, 2008, pp. 265-267.

WerLe, Gerhard. Principles of International Criminal Law. $3^{a}$ edición. Oxford, Oxford University Press, 2014, pp. $28-29$.

WerLe, Principles of International..., op.cit., p. 28; CASSESE et al., International Criminal Law..., op.cit., pp. 265-266. 
Revista Tribuna Internacional

Volumen $4 \cdot \mathrm{N}^{\circ} 8 \cdot 2015 \cdot$ pp. $271-282$

ISSN 0719-482X (versión en línea)

Luego de caracterizar al Tribunal Especial para El Líbano en particular, a continuación se analizará la resolución emitida el 21 de mayo de 2015 por la Sala de Juicio de ese tribunal sobre la admisibilidad de documentos publicados en el sitio web WikiLeaks, recaída sobre el caso "Ayyash et al." ".

\section{Naturaleza del tribunal}

Como se dijo, el Tribunal Especial para El Líbano pertenece al género denominado como "tribunales híbridos" o "internacionalizados". Este tipo de tribunales constituyen una novedad en el desarrollo del derecho penal internacional, cuya historia ha estado marcada principalmente por la creación de tribunales ad-hoc - v.g. el Tribunal Militar Internacional de Nüremberg de 1945, el Tribunal Militar Internacional para el Lejano Oriente de 1946, el Tribunal Penal Internacional para la ex Yugoslavia de 1993 y el Tribunal Penal Internacional para Ruanda de 1994- o completamente internacionales (como es el caso de la Corte Penal Internacional de 1998).

Por su parte, el Tribunal Especial para El Líbano no solamente se destaca por pertenecer a esta tendencia emergente en el desarrollo de la judicatura penal internacional, sino que presenta rasgos que lo vuelven en sí mismo triplemente excepcional en comparación a otros tribunales híbridos y a la judicatura penal internacional en general.

En primer lugar, su principal diferencia vis-à-vis los demás tribunales híbridos radica en que la jurisdicción ratione materiae del Tribunal Especial para El Líbano se reduce tan sólo al derecho penal libanés, en particular el tipo terrorista contenido en su Código Penal'. Por lo tanto, se trata de un tribunal híbrido tan sólo en su génesis o modo de origen, que no en su conocimiento y aplicación del derecho, pues su mandato lo vincula exclusivamente al derecho penal libanés. Este derecho define el terrorismo como "actos diseñados para crear un estado de alarma cometidos por medios tales como aparatos explosivos, materiales inflamables, productos venenosos o incendiarios o agentes microbiales capaces de crear un peligro público" (artículo 314 Código Penal de El Líbano). Dado que la ley no define qué actos reúnen estas características, la jurisprudencia libanesa ha entendido que los tribunales de ese país poseen discreción para determinar qué actos podrían ser subsumidos bajo este tipo penal, incluso llegando a presumir la intención especial terrorista a partir de ciertos hechos ${ }^{7}$. El peligro de ley

Tribunal Especial para El Líbano, Sala de Juicio, Fiscal v. Salim Jamil Ayyash, Mustafa Amine Badreddine, Hassan Habib Merbi, Hussein Hassan Oneissi and Assad Hassan Sabra (“Ayyash et al."), STL-11-01/T/TC, resolución del 21 de mayo de 2015.

6 JURDI, Nidal. "The Subject-Matter Jurisdiction of the Special Tribunal for Lebanon", en Journal of International Criminal Justice, $N^{\circ}$ 5, 2007, p. 1.126; BouнABIB, Melia. "Power and Perception: The Special Tribunal for Lebanon", en Berkeley Journal of Middle Eastern \& Islamic Law, vol. 3, No 1, 2010, p. 191.

7 JURDI, “The Subject-Matter...”, op.cit., pp. 1.129-1.134. 
penal en blanco que encierra esta tipificación y su aplicación en la práctica jurídica libanesa es manifiesto.

A pesar de lo anterior, en 2011 el Tribunal Especial se aventuró en la formulación de su propia definición del delito de terrorismo internacional en los siguientes términos:

"Un número de tratados, resoluciones de la ONU, y la práctica legislativa y judicial de los Estados evidencian la formación de una opinio iuris general en la comunidad internacional, acompañada de una práctica consistente con dicha opinión, en el sentido de que una norma consuetudinaria de derecho internacional relativa al crimen internacional de terrorismo, al menos en tiempos de paz, ha emergido. Esta regla consuetudinaria requiere los siguientes tres elementos fundamentales: (i) la perpetración de un acto criminal (como asesinato, secuestro, toma de rehenes, incendio, etc), o la amenaza de tal acto; (ii) la intención de difundir miedo entre la población (que en general conllevaría la creación de peligro público) o directa o indirectamente coaccionar a una autoridad nacional o internacional para adoptar una acción o abstenerse de ella; (iii) cuando el acto implica un elemento transnacional" (Resolución del derecho aplicable, 16 de febrero de 2011)

Sin embargo, a juicio de Kai Ambos el tercer requisito avanzado por el tribunal aún no se configura, debido a la falta de una declaración inequívoca de la comunidad internacional (por ejemplo, mediante un tratado que defina el delito de terrorismo, una declaración de la Asamblea General de las Naciones Unidas o una resolución de la Corte Internacional de Justicia) que permita establecer dicho "elemento transnacional". Para Ambos el terrorismo por el momento es tan sólo un treaty crime, pero admite que va en camino de convertirse en un crimen contra el derecho internacional propiamente tal ${ }^{9}$.

En segundo término, la referida génesis del Tribunal Especial del Líbano no gozó del desarrollo relativamente pacífico y consensuado de otras experiencias de negociación para el establecimiento de tribunales híbridos. Al contrario, luego de dos informes en que los enviados especiales de las Naciones Unidas atribuían abiertamente responsabilidades al gobierno de Siria por el atentado del 14 de febrero de 2005, la bancada pro Siria del Parlamento libanés y su presidente (también con afinidades hacia Siria) se negaron a ratificar el tratado internacional que su primer ministro había negociado con las Naciones Unidas. Por esta razón tuvo que intervenir el Consejo de Seguridad del organismo internacional para irrigar obligatoriedad jurídica al establecimiento del tribunal.

Se trató de la primera vez, en la breve historia de los tribunales híbridos, que se creó uno de ellos en aplicación de los poderes coercitivos que el Capítulo VII de la Carta de las Naciones Unidas entrega al Consejo de Seguridad, en lugar de ser el resultado de una negociación

CASSESE et al., International Criminal Law..., op.cit., pp. 148-149.

Ambos, Kai. Derecho Penaly Procesal Penal Internacionaly Europeo. Santiago, Thomson Reuters, 2013, pp. $159-162$. 
Revista Tribuna Internacional

Volumen $4 \cdot \mathrm{N}^{\text {o }} 8 \cdot 2015 \cdot$ pp. $271-282$

ISSN 0719-482X (versión en línea)

bilateral sin trabas ${ }^{10}$. Cabe señalar que en los comienzos de la historia de este tribunal, su constitución mediante los poderes coercitivos del Capítulo VII fue recibida con optimismo por parte de la doctrina, toda vez que se vislumbraba que su jurisprudencia podía gozar de fuerza vinculante internacional, en razón de su origen como órgano establecido en ejercicio de tales facultades ${ }^{11}$.

En tercer lugar, el Tribunal Especial para el Líbano también es singular debido a que su competencia ratione temporis se reduce a un hecho acaecido en el transcurso de un día -el atentado con bomba el 14 de febrero de 2005- y para poder conocer de otros hechos estos deben guardar alguna relación con ese acto concreto.

Por el contrario, los demás tribunales híbridos suelen definir su competencia temporal abarcando periodos de un año o mayor cantidad de tiempo, debido a que la masividad o sistematicidad de actos que "remecen la conciencia de la humanidad" y que suelen constituir la fuente material de este tipo de iniciativas, necesariamente requiere de cierto desarrollo a lo largo de un periodo importante de tiempo. En este sentido, la restricción temporal de la competencia del Tribunal Especial para El Líbano resulta tanto más incomprensible cuanto mayor es la cantidad de hechos que podrían revestir caracteres de delito ocurridos en la reciente historia de ese país, que incluye una guerra civil entre 1975 y 1990 y una guerra internacional con Israel en $2006^{12}$.

No obstante todas estas dificultades ontogenéticas y jurídicas, el tribunal comenzó su funcionamiento en 2009 y ha producido progresivamente un cuerpo de jurisprudencia digno de atención, del que forma parte la mencionada sentencia de 2011 que define el terrorismo como crimen internacional y aquella de 2015 que se pronuncia sobre la admisibilidad en juicio de documentos publicados en WikiLeaks, la que se analiza a continuación.

\section{Hechos del caso}

La causa Ayyash et al. se refiere al mencionado ataque perpetrado el 14 de febrero de 2005 en el Hotel Saint George en la ciudad de Beirut, que resultó en la muerte del ex Primer Ministro libanés Rafiq Hariri y de otras 22 personas.

En enero de 2011 la Fiscalía interpuso una acusación ante la Sala de Cuestiones Preliminares en contra de los individuos Salim Jamil Ayyash, Mustafa Amine Badreddine, Hussein Hassan Oneissi y Assad Hassan Sabra por el ataque de 2005, listado al que se agregó posteriormente el

\footnotetext{
BouнaвIB, "Power and Perception...", op.cit., pp. 175-188.

JURDI, “The Subject-Matter...", op.cit., p. 1.137.

BouHABIB, "Power and Perception...", op.cit., pp. 201-202.
} 
nombre de Hassan Habib Merhi. La Sala de Cuestiones Preliminares confirmó los cargos en junio de 2011 y emitió una orden de arresto para que las autoridades libanesas aprehendieran a los acusados.

El 26 de marzo de 2015 fue citado a declarar ante el tribunal el ex Primer Ministro de El Líbano (entre 2005 y 2009), el señor Fouad Siniora. Durante el contra-interrogatorio, la defensa del acusado Badreddine cuestionó al testigo por las circunstancias del establecimiento del Tribunal Especial para El Líbano, en 2007. Específicamente, la defensa lo interrogó acerca de una supuesta reunión sostenida el 6 de julio de 2007 entre el entonces Ministro de Justicia libanés y el embajador de Estados Unidos en ese país. El documento donde constaba la realización de dicha reunión había sido publicado en el sitio web WikiLeaks (medio de prueba de la defensa 1DT2-0312). El testigo negó tener conocimiento de dicha reunión y de cualquier acuerdo secreto entre El Líbano y Estados Unidos.

El 7 de mayo de 2015 la misma defensa interrogó al testigo llamado Walid Jumblatt, líder del Partido Socialista Progresista de El Líbano, acerca del radicalismo en ese país. En particular, la defensa le inquirió sobre una supuesta reunión con el embajador de Estados Unidos en El Líbano el 8 de abril de 2008, que también constaba en un documento publicado en WikiLeaks (medio de prueba de la defensa 2D133 $\mathrm{MFI}^{13}$ ).

En ambas oportunidades la Fiscalía objetó la admisibilidad de los documentos publicados en WikiLeaks como medios de prueba válidos, de conformidad al artículo 162(A) del Reglamento Procesal y de Prueba del Tribunal Especial ${ }^{14}$.

\section{Peticiones de las partes}

Al ser convocadas las partes a suscribir actuaciones referidas a la admisibilidad del primer documento, la defensa del acusado Badreddine sostuvo que aquél es relevante para sus argumentos, debido a que permite poner a prueba la credibilidad del ex Primer Ministro Siniora como testigo ${ }^{15}$.

En relación al valor probatorio de los documentos publicados en WikiLeaks, la defensa sostuvo que existe una tendencia emergente en la jurisprudencia internacional y comparada hacia la admisión de los mismos como medios de prueba ${ }^{16}$. En abono de su aserto la defensa

13 No obstante, no se generó ulterior debate en autos acerca de la admisibilidad de este segundo documento.

14 Artículo 162.- (A) No será admisible ningún medio de prueba que merezca dudas sustanciales en cuanto a su confiabilidad o cuya admisión fuese contraria a, y dañase severamente, la integridad del proceso.

(B) En particular, serán excluidos los medios de prueba que hayan sido obtenidos en contravención a los estándares internacionales de derechos humanos, incluyendo la prohibición de la tortura.

15 Solicitud por la parte Badreddine, párr.. 28.

16 Solicitud por la parte Badreddine, párrs. 24-26. 
Revista Tribuna Internacional

Volumen $4 \cdot \mathrm{N}^{\text {o }} 8 \cdot 2015 \cdot$ pp. $271-282$

ISSN 0719-482X (versión en línea)

citó la decisión del Tribunal Especial para Sierra Leona en el caso Charles Taylor, en el cual fue admitido como medio de prueba un cable del gobierno de Estados Unidos publicado en WikiLeaks $^{17}$. Asimismo, en el caso Gotovina, según la defensa, el Tribunal Penal Internacional para la ex Yugoslavia señaló que un "cable diplomático" gozaba de suficiente credibilidad 18 .

A mayor abundamiento, la defensa citó los casos Milosevic y Karadric y Milosevic, donde ni el Tribunal Internacional para la ex Yugoslavia ni el Mecanismo de las Naciones Unidas para Tribunales Penales Internacionales pusieron en duda la admisibilidad de documentos publicados en WikiLeaks ${ }^{19}$.

Por último, la defensa se refirió al caso Bancoult, en que el Alto Tribunal para Inglaterra y Gales resolvió que varios documentos de WikiLeaks podían ser usados para contra-interrogatorios ${ }^{20}$.

Por su parte, la Fiscalía respondió que no existe una tendencia jurisprudencial como la descrita por la defensa. Para ilustrar lo contrario, la Fiscalía se refirió al Caso 002 de las Cámaras Extraordinarias de los Tribunales de Camboya, en el cual se resolvió expresamente la inadmisibilidad de documentos publicados en WikiLeaks debido a su falta de autenticidad ${ }^{21}$.

En igual sentido, la Fiscalía se remitió a un caso fallado por la Corte del Distrito Federal de Estados Unidos (Unión Americana de Libertades Civiles c. Departamento de Estado), en el cual se resolvió que una publicación en WikiLeaks no cuenta como un reconocimiento oficial de parte de la autoridad ${ }^{22}$.

\section{Resolución del tribunal}

Tras examinar cada uno de los fallos citados por las partes, la Sala de Juicio decidió que la única sentencia que expresamente admitió como medios de prueba documentos publicados en WikiLeaks es aquella recaída en el caso Charles Taylor, aunque el Tribunal Especial para Sierra Leona no fundó su decisión ni analizó la autenticidad de los documentos ${ }^{23}$. En consecuencia,

17 Tribunal Especial para Sierra LeOna, Charles Taylor, SCSL-0301-T, Sala de Juicio II, resolución de 27 de enero de 2011, pp. 4-6.

18 Tribunal Penal Internacional para la ex Yugoslavia, IT-06-90-A, resolución de 2 de octubre de 2012 , párr. 26.

19 Tribunal Penal Internacional para la eX Yugoslavia, IT-02-54-Misc.5\&Misc.6, resolución de 18 de julio de 2011; Mecanismo de las Naciones Unidas para Tribunales Penales Internacionales, MiCT-13-55-R90.1 \& MiCT-1358-R90.1, resolución de 27 de noviembre de 2013.

20 Alto Tribunal de Inglaterra y Gales, Bancoult, EWHC 1502, resolución de 11 de junio de 2013, párrs. 27 y 35-36, [en línea] <http://www.bailii.org/ew/cases/EWHC/Admin/2013/1502.html> [consulta: 20.dic.2015]..

21 Cámaras Extraordinarias de los Tribunales de Camboya, Caso 002, E282, E282/1, E290, E290/1, resolución de 13 de junio de 2013, párrs. 7 y 11.

22 Corte Del Distrito Federal de Estados Unidos, 11-01072 (CKK), resolución del 23 de julio de 2012 , p. 10.

23 Tribunal Especial Para El Líbano, Ayyash et al., 21 de mayo de 2015, op.cit., párr. 29. 
no es efectivo que exista una "tendencia jurisprudencial" hacia la admisión en juicio de documentos publicados en WikiLeaks, como sostuvo la defensa ${ }^{24}$.

No obstante, la Sala de Juicio decidió seguir la doctrina del caso Bancoult resuelto en el Reino Unido. En esa oportunidad, el Alto Tribunal para Inglaterra y Gales admitió los contrainterrogatorios en relación al contenido vertido en los documentos de WikiLeaks, mas no en relación a dichos documentos per se $e^{25}$.

En relación a la admisibilidad de los documentos en sí mismos, la Sala de Juicio afirma que sigue con la doctrina establecida por la Corte Penal Internacional en Lubanga y por el Tribunal Penal Internacional para la ex Yugoslavia en Prlic et al., donde dichas cortes resolvieron que en la fase de admisibilidad no se requiere de prueba definitiva de confiabilidad y de autenticidad de los medios de prueba ofrecidos ${ }^{26}$.

Ahora bien, la Sala de Juicio afirmó que un medio de prueba debe cumplir con dos requisitos para poder ser declarado admisible: (i) relevancia para el argumento jurídico esgrimido en cada caso; y (ii) valor probatorio ${ }^{27}$.

El concepto de "valor probatorio" se desglosa en la noción de "confiabilidad", la cual a su vez se determina en función de los conceptos de "autenticidad” y "precisión"28.

En relación a la "confiabilidad" en general, se razona que debido a que la publicación de documentos en WikiLeaks constituye ya un hecho público y notorio, y a que el Departamento de Estado de Estados Unidos ha reconocido que algunos de ellos son cables oficiales filtrados, existe un "indicio de confiabilidad" en favor de los documentos publicados en esa página web $^{29}$.

Por otro lado, a falta de una admisión expresa de parte de alguna autoridad estatal en relación al carácter oficial de determinado documento subido a WikiLeaks, es imposible acreditar la "autenticidad" de los mismos.

Por último, en cuanto a la "precisión" de los documentos -es decir, su naturaleza no controvertida por otros medios de prueba- la Sala de Juicio determinó que el primer documento publicado en WikiLeaks (1DT2-0312), aún si fuese demostrado como auténtico, no es preciso debido a que fue controvertido por el testimonio rendido por el ex Primer Ministro de El Líbano ${ }^{30}$.

\footnotetext{
Tribunal Especial para El Líbano, Ayyash et al., 21 de mayo de 2015, op.cit., párr. 33.

5 Tribunal Especial para El Líbano, Ayyash et al., 21 de mayo de 2015, op.cit., párrs. 34 y 43.

26 Aunque la Sala de Juicio reconoce que la Corte Penal Internacional adoptó una postura exactamente opuesta a la mencionada en Katanga, donde excluyó prima facie aquellos medios no demostrados desde el inicio como relevantes y auténticos. Tribunal Especial Para El Líbano, Ayyash et al., 21 de mayo de 2015, op.cit., párrs. 11-12.

27 Tribunal Especial Para El Líbano, Ayyash et al., 21 de mayo de 2015, op.cit., párrs. 11-12.

28 Tribunal Especial Para El Líbano, Ayyash et al., 21 de mayo de 2015, op.cit., párr. 34.

29 Tribunal Especial para El Líbano, Ayyash et al., 21 de mayo de 2015, op.cit., párr. 36.

30 Tribunal Especial para El Líbano, Ayyash et al., 21 de mayo de 2015, op.cit., párr. 42.
} 
Revista Tribuna Internacional

Volumen $4 \cdot \mathrm{N}^{\text {o }} 8 \cdot 2015 \cdot$ pp. $271-282$

ISSN 0719-482X (versión en línea)

Por todas estas consideraciones, mediante resolución del 21 de mayo de 2015, la Sala de Juicio declaró inadmisibles como medios de prueba los documentos publicados en WikiLeaks, si bien permitió la realización de contra-interrogatorios acerca del contenido de los mismos.

El 28 de mayo de 2015 la defensa del acusado Badreddine presentó ante la misma Sala de Juicio una solicitud para obtener certificación para apelar en contra de la decisión, de conformidad a lo dispuesto en el artículo 126(C) del Reglamento del Tribunal ${ }^{31}$. La Sala de Juicio señaló que el umbral de apelación establecido en dicho reglamento es bastante alto, por lo que el recurso solamente se concede de manera excepcional debido a consideraciones de "economía procesal"32.

En definitiva, la Sala de Juicio denegó la certificación para apelar, debido a que los documentos que se intentaron ofrecer como medios de prueba poseían "relevancia, cuando mucho, marginal" para el proceso ${ }^{33}$. Sin embargo, admitió que su resolución de 21 de mayo se refiere tan sólo a la (falta de) autenticidad de dos documentos de WikiLeaks en particular, sin negarse en principio a que otros documentos subidos al sitio web puedan constituir, en sus propios méritos, medios de prueba admisibles en juicio ${ }^{34}$.

\section{Análisis}

El Tribunal Especial para El Líbano verificó en su sentencia del 21 de mayo de 2015 que no es correcto que exista una "tendencia jurisprudencial" en el derecho internacional y comparado hacia la admisibilidad de documentos de WikiLeaks como medios de prueba en juicio. Para ello el Tribunal tuvo a la vista la existencia de tres sentencias fundamentales: una favorable a esta supuesta tendencia, otra contraria y una que procura conferirles a estos documentos el mayor rendimiento argumentativo posible.

En efecto, la principal sentencia citada por la defensa es aquella pronunciada por el Tribunal Especial para Sierra Leona en el caso Charles Taylor en 2011. En aquella oportunidad la defensa del ex dictador de Liberia solicitó la introducción intempestiva de cables publicados en WikiLeaks como medios de prueba favorables al acusado (pues pondrían en duda la imparcialidad del tribunal para procesarlo), los cuales fueron liberados en el sitio web luego del

\footnotetext{
31 Artículo 126.- [...] (C) [La Sala de Juicio debe certificar una solicitud de apelación] si la decisión involucra un asunto que pudiese afectar significativamente la conducción justa y expedita del procedimiento o de las resultas del juicio, y para la cual una decisión inmediata de la Cámara de Apelaciones pudiese impulsar sustantivamente el proceso.

32 Tribunal Especial para El Líbano, Ayyash et al., 3 de julio de 2015, op.cit., párr. 5.

33 Tribunal Especial para El Líbano, Ayyash et al., 3 de julio de 2015, op.cit., párr. 9.

34 Tribunal Especial para El Líbano, Ayyash et al., 3 de julio de 2015, op.cit., párr. 8.
} 
pronunciamiento de la sentencia de la Segunda Sala de Juicio. La Sala de Juicio resolvió acoger la petición, citando lo resuelto anteriormente por la Sala de Apelaciones, la cual había accedido a

“[...] 'permitir la recepción de información -aseveraciones de hecho (pero no opiniones) hechas en documentos o comunicaciones electrónicas- si es que tales hechos son relevantes y si es que su confiabilidad es susceptible de confirmación'. Esta fraseología fue escogida para dejar en claro que la prueba de confiabilidad no es una condición de admisibilidad: todo lo que se exige es que la información debería ser susceptible de corroboración a su debido tiempo"35.

Como se puede observar, no es efectivo que el Tribunal para Sierra Leona no haya fundado su resolución -como lo sostiene el Tribunal para El Líbano- sino que aquél citó la doctrina de la Sala de Apelaciones (que replica aquella de la Corte Penal Internacional en Lubanga y la del Tribunal Penal Internacional para la ex Yugoslavia en Prlic et al.) que consiste en que la confiabilidad de un medio de prueba no debe ser resuelta in limine como requisito para que éste pueda ser declarado al menos como un medio admisible.

Por el contrario, la sentencia más importante citada por la Fiscalía del Tribunal Especial para El Líbano en Ayyash et al. es aquella pronunciada por las Cámaras Extraordinarias para los Tribunales de Camboya en el Caso 002 en 2013, las cuales sostuvieron que "todos los cables diplomáticos propuestos provenientes del sitio web WikiLeaks no son, oficialmente, del Departamento de Estado norteamericano". En ese mismo orden de ideas, la Cámara de primera instancia finalmente resolvió que

“(...) a primera vista, los medios de prueba propuestos no cumplen con los criterios de pertinencia y de confiabilidad (incluyendo en relación a la autenticidad), de conformidad a la regla 87. 3) del Reglamento Interno y rechaza la solicitud de los co-procuradores y de la defensa de KHIEU Samplan en razón de la imposibilidad de obtener las copias certificadas conformes de los cables diplomáticos en cuestión dentro de un plazo razonable" 36 .

Por último, otro fallo citado por la defensa en Ayyash et al. y seguido por el Tribunal Especial para El Líbano es aquel recaído sobre la causa Bancoult, pronunciado por el Alto Tribunal para Inglaterra y Gales en 2013. Luego de permitir que durante los procedimientos un abogado interrogase a un testigo acerca de los contenidos de un documento publicado en WikiLeaks, los miembros del Alto Tribunal afirmaron que "no necesitamos indagar sobre la autenticidad del documento. Simplemente asumiremos ad arguendum ['for the purposes of argument] que es genuino. Si lo es, sabemos poco acerca del mismo además de su contenido".

5 Tribunal Especial para Sierra LeOnA, Charles Taylor, 27 de enero de 2011, op.cit., pp. 4-6.

36 Cámaras Extraordinarias de los Tribunales de Camboya, E282, E282/1, E290, E290/1, resolución de 13 de junio de 2013, párrs. 7, 11. 
Revista Tribuna Internacional

Volumen $4 \cdot \mathrm{N}^{\text {o }} 8 \cdot 2015 \cdot$ pp. $271-282$

ISSN 0719-482X (versión en línea)

Incluso aceptando la posibilidad de que el documento hubiese sido obtenido de manera ilícita -pereat la teoría del "fruto del árbol envenenado"- el Alto Tribunal finalmente decidió que

“[...] Estamos conformes con mitigar la preocupación mediante la adopción del criterio que ya hemos señalado, de asumir que el documento es genuino, sin averiguar si de hecho lo es.

[...] La extensa divulgación previa del documento y de la información contenida en él implica que una ulterior divulgación realizada mediante su uso en este procedimiento no es dañina. Si no se comete ningún delito, el hecho de que, en la primera divulgación, se pueda haber cometido un delito por alguna persona no debería prevenir su uso en este procedimiento" ${ }^{37}$.

La doctrina sustraída del caso Bancoult procura conferir rendimiento argumentativo en juicio a un documento -incluso si fue obtenido por medios originalmente delictivos o ilícitos- al permitir a los representantes de las partes interrogar a los testigos acerca del contenido de dichos documentos. Esta doctrina, seguida prudencialmente por el Tribunal Especial para El Líbano en el fallo en comento, parece saludable para el respeto del derecho a la defensa y de los derechos humanos en general durante la conducción de los procesos que en el marco de la "guerra contra el terror" se han seguido a lo largo de todo el mundo desde 2001.

En efecto, la Asamblea General de las Naciones Unidas definió su "Estrategia Global para la Lucha contra el Terrorismo" en $2006^{38}$, la cual establece la necesidad de respetar los derechos humanos mientras se combate este flagelo mundial. En particular, la Estrategia ordena que los Estados "deben asegurarse de que toda medida adoptada para combatir el terrorismo esté de acuerdo a sus obligaciones bajo el derecho internacional, en particular con el derecho de los derechos humanos, de los refugiados y el derecho internacional humanitario" (Título IV, párr. 2).

Por lo tanto, siendo El Líbano miembro de las Naciones Unidas, y atendida la naturaleza híbrida del Tribunal Especial para ese país, las resoluciones emitidas por la Sala de Juicio en el caso Ayyash et al. deben ser evaluadas desde una perspectiva de derechos humanos, sobre todo considerando que se trata de un tribunal establecido por motivos eminentemente políticos en el contexto de la lucha internacional contra el terrorismo.

En tal sentido, lo positivo de las resoluciones recaídas en Ayyash et al. consiste en que, si bien no se admiten per se los documentos publicados en WikiLeaks como medios de prueba, se permite interrogar a los testigos en relación al contenido de los mismos, lo cual amplía las posibilidades de defensa de los procesados por delitos terroristas.

Asimismo, es digno de encomio el hecho de que en su segunda resolución la Sala de Juicio se haya mostrado abierta a declarar admisibles en el futuro este tipo de documentos como medios de prueba. Cabe señalar, no obstante, que el peligro radica en que la Fiscalía podría aprovechar

37 Alto Tribunal de InglaterRa y Gales, Bancoult, 11 de junio de 2013, op.cit., párrs. 27 y 35-36.

38 Asamblea General de las NAciones Unidas, Resolución 60/288 de 20 de septiembre de 2006. 
esta decisión para presentar medios de prueba poco confiables del mismo sitio WikiLeaks, en su celo por obtener condenas, lo cual socavaría el principio del mayor peso de la prueba que ésta debería cargar en sistemas donde impera la presunción de inocencia en materia criminal.

Ahora bien, hablando en términos más generales, la decisión de la Sala de Juicio atenta en contra de los derechos humanos de los acusados, en particular en lo referente al derecho al debido proceso, al no haber concedido la apelación solicitada. Si bien es el propio Reglamento del Tribunal el que restringe sobremanera el derecho a apelar, lo cierto es que la Sala de Juicio hace suyas las razones para el establecimiento de tal regulación, aduciendo consideraciones de "economía procesal", en circunstancias que pudo haber guardado silencio acerca del mérito de dicha regulación o haberla criticado. Esto podría parecer improbable atendido que son los propios miembros del Tribunal quienes redactan dicho reglamento, pero siempre hay espacio, a lo menos, para opiniones disidentes dentro de una magistratura colegiada.

No se debe olvidar que el derecho al debido proceso, que incluye la posibilidad no nugatoria de interponer un recurso de apelación ante un tribunal superior, es una de las garantías que la jurisprudencia internacional de derechos humanos ha declarado debe ser respetada aún en el fragor de la "guerra contra el terror". Esto puede observarse en lo resuelto en los últimos años por la Corte Interamericana de Derechos Humanos en Norín Catrimán y otros c. Chile 39 $^{39}$ por la Corte Europea de Derechos Humanos en el caso Egamberdiyev ${ }^{40}$ y por el Tribunal de Justicia de la Unión Europea en los casos Kadi I y Kadi II ${ }^{41}$.

Es de esperar que la doctrina Bancoult permita en el futuro a éste y a otros tribunales domésticos e internacionales considerar toda la evidencia puesta a su disposición de la manera más imparcial y respetuosa del principio de presunción de inocencia posible, para que la comunidad jurídica internacional pueda determinar a cabalidad y agotando siempre el principio deliberativo ad arguendum si es que la fuerza del ius puniendi nacional o internacional ha de caer sobre los acusados.

39 Corte Interamericana de Derechos Humanos, Norín Catrimán y otros c. Chile, resolución de 29 de mayo de 2014 , párrs. 267-270.

40 Corte Europea de Derechos Humanos, Egamberdiyev c. Rusia, resolución de 26 de junio de 2014 , párr. 53.

41 Tribunal de Justicia de la Unión EuropeA, Kadi y Al Barakaat International Foundation c. Consejo y Comisión, resolución de 3 de septiembre de 2008, párr. 344; Comisión y otros c. Kadi, resolución de 18 de julio de 2013, párr. 125. 